\title{
BMJ Open IMAGINE Network's Mind And Gut Interactions Cohort (MAGIC) Study: a protocol for a prospective observational multicentre cohort study in inflammatory bowel disease and irritable bowel syndrome
}

Paul Moayyedi (D) , ${ }^{1}$ Glenda MacQueen, ${ }^{2}$ Charles N Bernstein, ${ }^{3}$ Stephen Vanner, ${ }^{4}$ Premysl Bercik, ${ }^{1}$ Karen L Madsen, ${ }^{5}$ Michael Surette, ${ }^{6}$ John D Rioux, ${ }^{7}$ Levinus A Dieleman, ${ }^{8}$ Elena Verdú, ${ }^{9}$ Russell $\mathrm{J}$ de Souza, ${ }^{10}$ Anthony Otley, ${ }^{11}$ Laura Targownik, ${ }^{12}$ John Lavis, ${ }^{6}$ Jennifer Cunningham (D) , ${ }^{13}$ Deborah A Marshall (D) ${ }^{14}$ Sandra Zelinsky, ${ }^{15}$ Aida Fernandes ${ }^{1}$

To cite: Moayyedi $P$, MacQueen G, Bernstein CN, et al. IMAGINE Network's Mind And Gut Interactions Cohort (MAGIC) Study: a protocol for a prospective observational multicentre cohort study in inflammatory bowel disease and irritable bowel syndrome. BMJ Open 2020;10:e041733. doi:10.1136/ bmjopen-2020-041733

- Prepublication history for this paper is available online. To view these files, please visit the journal online (http://dx.doi org/10.1136/bmjopen-2020041733).

Received 18 June 2020 Revised 05 September 2020 Accepted 22 September 2020

Check for updates

(C) Author(s) (or their employer(s)) 2020. Re-use permitted under CC BY-NC. No commercial re-use. See rights and permissions. Published by BMJ.

For numbered affiliations see end of article.

Correspondence to Professor Paul Moayyedi; moayyep@mcmaster.ca

\section{ABSTRACT}

Introduction Gut microbiome and diet may be important in irritable bowel syndrome (IBS), inflammatory bowel disease (IBD) and comorbid psychiatric conditions, but the mechanisms are unclear. We will create a large cohort of patients with IBS, IBD and healthy controls, and follow them over time, collecting dietary and mental health information and biological samples, to assess their gastrointestinal (Gl) and psychological symptoms in association with their diet, gut microbiome and metabolome.

Methods and analysis This 5-year observational prospective cohort study is recruiting 8000 participants from 15 Canadian centres. Persons with IBS who are 13 years of age and older or $\mathrm{IBD} \geq 5$ years will be recruited. Healthy controls will be recruited from the general public and from friends or relatives of those with IBD or IBS who do not have Gl symptoms. Participants answer surveys and provide blood, urine and stool samples annually. Surveys assess disease activity, quality of life, physical pain, lifestyle factors, psychological status and diet. The main outcomes evaluated will be the association between the diet, inflammatory, genetic, microbiome and metabolomic profiles in those with IBD and IBS compared with healthy controls using multivariate logistic regression. We will also compare these profiles in those with active versus quiescent disease and those with and without psychological comorbidity.

Ethics and dissemination Approval has been obtained from the institutional review boards of all centres taking part in the study. We will develop evidence-based knowledge translation initiatives for patients, clinicians and policymakers to disseminate results to relevant stakeholders.

Trial registration number: NCT03131414

\section{INTRODUCTION}

Two-thirds of the population experience significant gastrointestinal (GI) symptoms at some point in their life. ${ }^{1}$ One of the most common GI disorders is irritable bowel syndrome (IBS),

\section{Strengths and limitations of this study}

- This is the largest observational study evaluating the microbiome in inflammatory bowel disease and irritable bowel syndrome.

- The patients' disease type and activity are well characterised with detailed information on diet and mental health.

- The degree of patient engagement is another strength of the study.

- The microbiome and diet assessment are conducted once per year and may not correlate with disease flare-ups.

- As with all observational studies, any association may not be causal and will need evaluation in randomised controlled trials.

affecting up to $10 \%$ of persons worldwide, depending on the definition. ${ }^{2}$ Another GI disorder that is associated with significant healthcare resources is inflammatory bowel disease (IBD) which affects approximately $0.3 \%$ of the world's industrialised population. ${ }^{3}$

The cardinal features of IBS include chronic abdominal pain over 3 months per year related to a change in bowel habit and the disease can present at any age. ${ }^{24}$ IBD is a term encompassing two distinct but related diseases: ulcerative colitis (UC) and Crohn's disease (CD). The cardinal symptom of UC is bloody diarrhoea while in CD abdominal pain is a more prominent symptom, but diarrhoea is a feature as well. ${ }^{5}$ IBD has the highest incidence in second and third decades of life and are lifelong relapsing and remitting 
diseases. Growth impairment can be an issue in children with CD.

Both IBS and IBD significantly impact quality of life ${ }^{67}$ and often surgery is needed in patients with IBD. ${ }^{8}$ IBS and IBD also account for significant healthcare spending in the developed world with many countries spending billions of dollars per annum. ${ }^{9}$ Persons with IBS and IBD have higher rates of anxiety and depression compared with the general population ${ }^{11}{ }^{11}$ and those with other chronic diseases. ${ }^{12}$ The corollary is also true; persons with anxiety and depression have more GI symptoms compared with healthy controls. ${ }^{13}$ Therapy for IBS has traditionally focused on drugs that alter motility or visceral sensitivity of the GI tract and although various interventions are superior to placebo, the overall impact on symptoms is only modest. ${ }^{14}$ Therapy for IBD has traditionally focused on drugs that inhibit the exaggerated pro-inflammatory immune response, however only $50 \%$ of the patients achieve clinical remission, whereas clinical relapses are common.

There is evidence that the gut microbiome and diet are important in IBS, ${ }^{15} \mathrm{IBD}^{16}$ and comorbid psychiatric conditions. ${ }^{17}$ There is however a need for more longitudinal prospective data on this interaction in patients with IBD and IBS compared with healthy controls. We have conducted systematic reviews in both $\mathrm{IBS}^{18}$ and $\mathrm{IBD}^{19}$; although there are numerous case control studies exploring the gut microbiome in these conditions, the median sample size is around 20 per arm and in all cases the sample size was insufficient to deal with the multiple testing issues that relate to microbiome research in humans. In addition, inter-individual differences of the gut microbiome are large. Large sample sizes and longitudinal sampling within the same individuals over time are therefore needed to evaluate the interaction between diet, the microbiome, IBS, IBD and associated mental health issues.

The Inflammation, Microbiome and Alimentation: GastroIntestinal and Neuropsychiatric Effects (IMAGINE) ${ }^{20}$ (see online supplemental appendix 1) Strategy for Patient Oriented Research (SPOR) Network is conducting a 5-year multicentre prospective observational cohort study, Mind And Gut Interactions Cohort (MAGIC). It will explore the interaction between the diet, microbiome and the host associated with IBS and IBD in order to better target treatment of IBD and IBS and the psychiatric disorders associated and affecting these diseases. The overarching hypothesis of this study is that IBS and IBD are driven by a perturbation of the gut microbiome and the associated host immune response. Alterations in the gut microbiome may also drive anxiety and depression associated with these GI disorders and these psychological factors may in turn influence gut symptoms and its microbiome. These mechanisms may also have a genetic predisposition.

\section{Aims}

The main aim of the MAGIC Study conducted through the IMAGINE SPOR Network is to create a large cohort of patients with IBS, IBD and healthy controls and follow these individuals over time, assessing disease activity, diet, mental health and demographic information using validated questionnaires and collecting annual stool, urine and blood samples, to correlate GI and psychological symptoms with an individual's genetic variants, diet and gut microbiome, as well as host and microbiome metabolic products in stool, urine and serum.

\section{Primary aims for baseline data}

1. We will compare the gut microbiome and metabolomic profile between $\mathrm{CD}$, UC, IBS and healthy controls. The main analyses will be between a specific disorder and healthy controls.

2. We will compare the gut microbiome and metabolomic profile of participants with active versus quiescent disease within CD, UC and IBS.

3 . We will compare the gut microbiome and metabolomic profile of participants with and without psychiatric comorbidity for each of $\mathrm{CD}, \mathrm{UC}$, IBS and healthy controls.

\section{Primary aims for longitudinal data}

1. Microbiome, metabolomic, genetic, inflammatory markers, dietary, disease phenotype, psychiatric comorbidity and demographic predictors of failure of therapy for each of UC, CD and IBS.

2. Comparison of microbiome, metabolomic, genetic, dietary and demographic factors in IBD of participants who remain in clinical remission over 2 years versus those with recurrent active disease (UC and CD analysed separately).

3. Comparison of gut microbiome, metabolomic, genetic, dietary and demographic factors in participants with IBS with mild/inactive disease (based on IBS-Symptom Severity Score (SSS) ) compared with those with ongoing active disease. IBS will be evaluated overall and also within subgroups independently.

\section{Secondary aims for baseline data}

1. To compare dietary patterns between patients with $\mathrm{CD}$, UC, IBS and healthy controls.

2. To compare genetic risk factors between $\mathrm{CD}, \mathrm{UC}$, IBS and healthy controls.

3. To compare gut microbiome, metabolomic, genetic, dietary and demographic factors in participants with IBD with quiescent inflammatory disease with and without concomitant IBS (defined by faecal calprotectin $<50 \mu \mathrm{g} / \mathrm{g}$ and subthreshold IBD symptom activity score but who have active IBS symptoms on IBS-SSS).

4. To compare the prevalence of mood and anxiety disorders in participants with each disorder against rates in healthy controls.

5. To compare the dietary, gut microbiome and metabolomic profile between participants with or without anxiety (CD, UC, IBS, healthy controls analysed separately).

6. To compare the dietary, gut microbiome and metabolomic profiles between participants with and without 
depression (CD, UC, IBS, healthy controls analysed separately).

7. To determine whether high rates of early adverse experiences are associated with history of more severe disease or treatment resistance in participants with $\mathrm{CD}$, UC or IBS.

8. To examine the association between symptom severity and multiple domains of function in participants with CD, UC or IBS.

9. To develop models describing how factors such as microbiome, metabolome, diet, genes and psychiatric symptoms interact in CD, UC and IBS.

\section{Secondary aims for longitudinal data}

1. To compare healthcare resource use between CD, UC, IBS and healthy controls.

2. To compare work productivity between $\mathrm{CD}$, UC, IBS and healthy controls.

3. To compare baseline dietary and gut microbiome and urinary metabolome profiles and inflammatory markers in participants that develop anxiety during followup versus those who did not have anxiety at any time (CD, UC, IBS and healthy controls analysed separately).

4. To compare baseline dietary and gut microbiome and urinary metabolome profiles in participants that develop depression during follow-up versus those that did not have depression at any time (CD, UC, IBS and healthy controls analysed separately and if appropriate combined).

5. To compare gut microbiome, urinary metabolome, genetic, dietary and demographic factors in IBS at baseline in those that change their IBS subtype during follow-up and those that continue with the same IBS subgroup.

6. To compare which dietary, mental health, gut microbiome and metabolomic profiles precede a clinical relapse in patients with IBD and how these parameters are different in patients with active disease versus those who remain in clinical remission.

\section{METHODS \\ Design}

The IMAGINE MAGIC Study is a prospective observational cohort study that is recruiting 2000 participants with each of CD, UC and IBS and also 2000 healthy participants in 15 centres across Canada. Assessment includes psychological status, dietary intake, gut microbiome, urinary metabolomic profile, inflammatory markers, genotype, health-related quality of life, and healthcare resource use and associated costs. The cohort and healthy controls will be followed annually for up to 4 years after the baseline study enrolment.

At each visit, the participant provides blood, urine and stool samples, as well as complete questionnaires assessing disease activity, quality of life, physical pain, lifestyle factors, psychological status and diet. Table 1 summarises participant information collected at each visit.

\section{Participants}

A total of 8000 participants will be recruited, 2000 for each for healthy volunteers, IBS, UC and CD. Inclusion and exclusion criteria are outlined in table 1.

Healthy participants over the age of 4 years will be recruited from the relatives, spouses and friends of IBS and IBD cases taking part and also through advertisement for healthy volunteers. Patients with IBS and IBD will primarily be recruited from gastroenterology clinics at participating centres.

\section{Irritable bowel syndrome}

Persons who meet Rome IV criteria and are 13 years of age or older are enrolled (table 1). Persons with IBS are categorised into diarrhoea-predominant IBS (IBS-D), constipation-predominant IBS (IBS-C), IBS with mixed bowel habits (IBS-M) or unclassified IBS. ${ }^{21}$ Patients with IBS that also have coeliac disease will be eligible provided they continue to have symptoms after 6 months of a gluten-free diet and their tissue transglutaminase antibody has returned to normal.

\section{Inflammatory bowel disease}

Persons with either CD or UC over the age of 4 years are enrolled regardless of whether the disease is active or in remission (table 1). Persons with unclassified IBD are included. The Montreal classification is used for adult patients with $\mathrm{CD}$ and $\mathrm{UC},{ }^{22}$ and the Paris classification $^{23}$ for paediatric IBD. The research coordinator conducts a chart review to confirm the date of diagnosis and maximal phenotype at time of enrolment using the Montreal classification.

\section{Data collection}

All participants attend a baseline, 12-month, 24-month, 36-month and 48-month visit. Sources of information for each patient are blood, urine and stool samples, questionnaires and chart review (table 2).

Participants complete a questionnaire to obtain age, sex, gender identity, education level attained, ethnic heritage, smoking/alcohol/drug history, comorbidities, medication and therapies, menstrual status at baseline. The study research coordinator records height, weight, body mass index (BMI) and disease-related information. ${ }^{24}$ Participants answer a series of validated questionnaires to assess disease activity, quality of life, physical pain, lifestyle factors, psychological status and diet at each study visit (table 3). Questionnaires measuring anxiety, depression, generalised anxiety disorder, sleep disturbance, perceived stress, adverse childhood experiences, resiliency and pain catastrophising in both adults and children are outlined in table 3.

Healthcare resource uses data and associated costs regarding physician visits, clinical procedures, imaging procedures, hospitalisations, emergency room visits, and medication use are collected by linking the participants 


\begin{tabular}{|c|c|c|}
\hline Type & Inclusion criteria & Exclusion criteria \\
\hline IBD & $\begin{array}{l}\text { Patients with documented CD, UC or IBD-U, }>4 \text { years } \\
\text { old }\end{array}$ & $\begin{array}{l}\text { Patients with subtotal colectomy and/or ileostomy } \\
\text { Major comorbid condition where the projected survival is } \\
\text { less than } 5 \text { years } \\
\text { Difficulties with communication, including unable to } \\
\text { communicate in English or French } \\
\text { Diagnosis of schizophrenia } \\
\text { Diagnosis of eating disorder }\end{array}$ \\
\hline IBS & $\begin{array}{l}\text { Patients with IBS who have met Rome IV criteria, } \geq 13 \\
\text { years old } \\
\text { IBS-D } \\
\text { Normal CBC } \\
\text { Negative tissue transglutaminase antibody if } \\
\text { diarrhoea the main symptom } \\
\text { Symptoms onset }>45 \text { years old, then negative } \\
\text { colonic biopsies for microscopic colitis } \\
\text { IBS-C, IBS-M and IBS-U } \\
\text { Negative tissue transglutaminase antibody } \\
\text { Symptoms onset }>50 \text { years age, with new symptoms } \\
<1 \text {-year duration, then have a negative colonoscopy, } \\
\text { CT colonography or Air Contrast Barium Enema } \\
\text { Normal CBC }\end{array}$ & $\begin{array}{l}\text { Major Gl surgery (Roux en y, bowel resection) } \\
\text { Major comorbid condition, where the projected survival is } \\
\text { less than } 5 \text { years } \\
\text { Drug use that is the major cause of Gl symptoms and/ } \\
\text { or undermines longitudinal compliance, including chronic } \\
\text { antibiotic use, narcotic analgesics and substance abuse } \\
\text { Narcotic analgesic use causing Gl symptoms } \\
\text { Difficulties with communication, including unable to } \\
\text { communicate in English or French } \\
\text { Diagnosis of schizophrenia } \\
\text { Diagnosis of eating disorder } \\
\text { Gl cancer within } 5 \text { years }\end{array}$ \\
\hline Healthy controls & No Gl symptoms using the Rome IV Questionnaire & $\begin{array}{l}\text { Major Gl surgery (Roux en y, bowel resection) } \\
\text { Any major comorbid chronic condition } \\
\text { Difficulties with communication, including unable to } \\
\text { communicate in English or French } \\
\text { Diagnosis of schizophrenia } \\
\text { Diagnosis of eating disorder }\end{array}$ \\
\hline
\end{tabular}

CBC, complete blood count; CD, Crohn's disease; GI, gastrointestinal; IBD, inflammatory bowel disease; IBD-U, unclassified IBD; IBS, irritable bowel syndrome; IBS-C, constipation-predominant IBS; IBS-D, diarrhoea-predominant IBS; IBS-M, IBS with mixed bowel habits; IBS-U, unclassified IBS; UC, ulcerative colitis.

to provincial administrative data from the Canadian Gastro-Intestinal Epidemiology Consortium (https:// cangiec.ca).

\section{Biosamples}

A stool sample is collected for faecal microbiome, inflammatory markers and short-chain fatty acids. A urine sample is collected for metabolomics, and blood samples are taken for DNA isolation as well as for serum for inflammatory markers (ie, cytokines, chemokines, high-sensitivity $\mathrm{C}$ reactive protein, lipopolysaccharide) and metabolomic profile (eg, tryptophan metabolites, growth factors such as brain-derived neurotrophic factor, neurotransmitters such as GABA and serotonin, and stress hormones such as cortisol) at each study visit. All biosamples are shipped to Population Health Research Institute (PHRI) for storing at $-80^{\circ} \mathrm{C}$.

\section{Data management}

Questionnaires are completed electronically using tablets during the clinic visit with the option to complete the any questionnaires remaining after clinic visit at home on a

\begin{tabular}{|c|c|c|c|c|c|c|}
\hline Contact & Screening pe & riod & 12-month visit & 24-month visit & 36-month visit & 48-month visit \\
\hline Purpose & Enrol in study & & $\begin{array}{l}\text { Health status } \\
\text { Biosamples } \\
\text { Questionnaires }\end{array}$ & $\begin{array}{l}\text { Health status } \\
\text { Biosamples } \\
\text { Questionnaires }\end{array}$ & $\begin{array}{l}\text { Health status } \\
\text { Biosamples } \\
\text { Questionnaires }\end{array}$ & $\begin{array}{l}\text { Health status } \\
\text { Biosamples } \\
\text { Questionnaires }\end{array}$ \\
\hline $\begin{array}{l}\text { Information } \\
\text { collected }\end{array}$ & $\begin{array}{l}\text { Contact by } \\
\text { telephone, } \\
\text { email or clinic } \\
\text { visit. } \\
\text { Explain } \\
\text { project } \\
\text { Set up study } \\
\text { visit(s) }\end{array}$ & $\begin{array}{l}\text { Signed consent } \\
\text { Eligibility } \\
\text { screening } \\
\text { Demographics } \\
\text { Medical history } \\
\text { Provide urine and } \\
\text { stool kit } \\
\text { Obtain blood } \\
\text { (+DNA) } \\
\text { Questionnaires }\end{array}$ & $\begin{array}{l}\text { Medical history } \\
\text { Obtain urine, } \\
\text { stool, blood } \\
\text { Questionnaires }\end{array}$ & $\begin{array}{l}\text { Medical history } \\
\text { Obtain urine, } \\
\text { stool, blood } \\
\text { Questionnaires }\end{array}$ & $\begin{array}{l}\text { Medical history } \\
\text { Obtain urine, } \\
\text { stool, blood } \\
\text { Questionnaires }\end{array}$ & $\begin{array}{l}\text { Medical history } \\
\text { Obtain urine, } \\
\text { stool, blood } \\
\text { Questionnaires }\end{array}$ \\
\hline
\end{tabular}


Table 3 List of patient-answered questionnaires

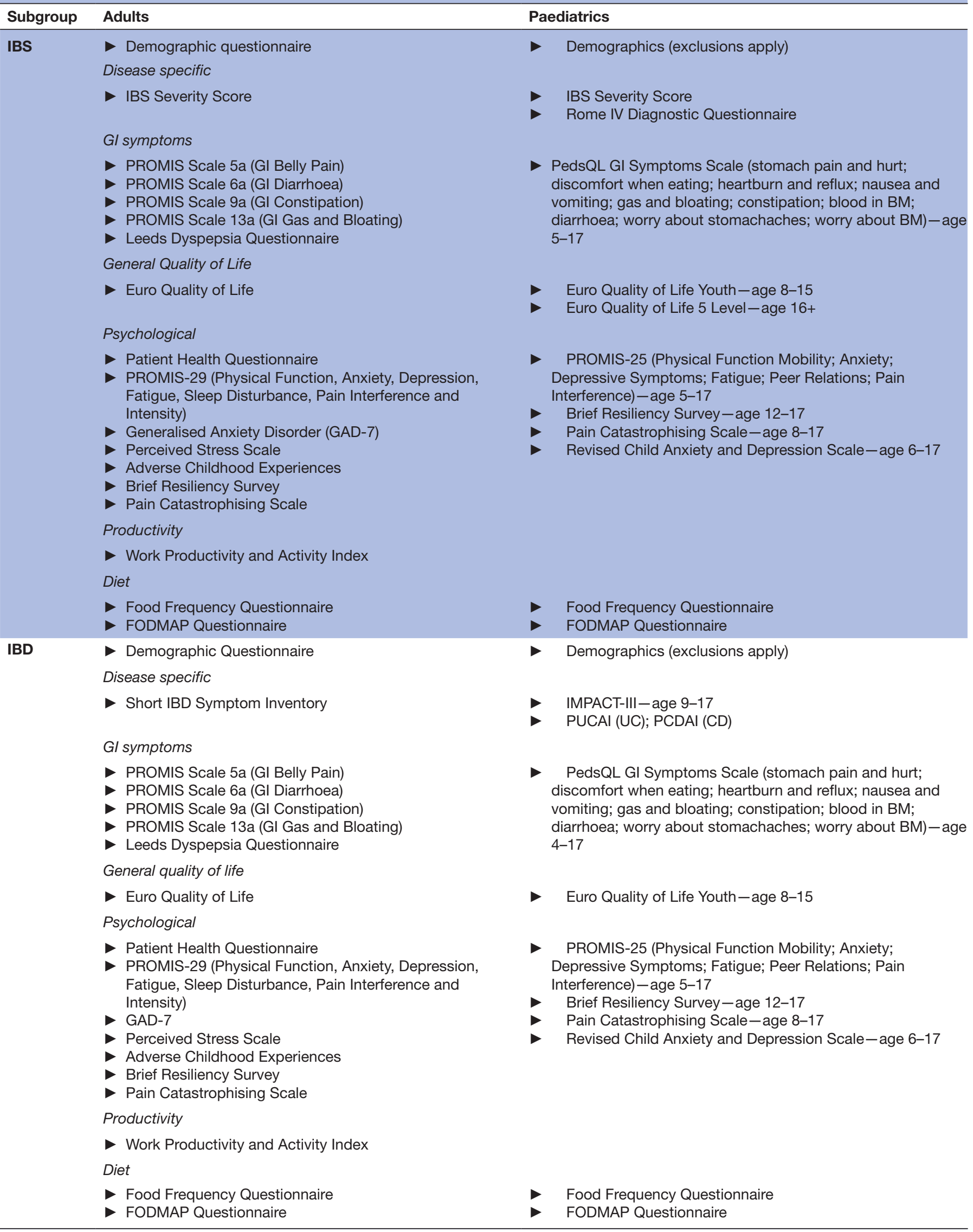




\begin{tabular}{|c|c|c|}
\hline Subgroup & Adults & Paediatrics \\
\hline \multirow[t]{14}{*}{$\begin{array}{l}\text { Healthy } \\
\text { controls }\end{array}$} & $\begin{array}{l}\text { Demographic Questionnaire } \\
\text { Gl symptoms }\end{array}$ & Demographics (exclusions apply) \\
\hline & $\begin{array}{l}\text { PROMIS Scale 5a (GI Belly Pain) } \\
\text { PROMIS Scale 6a (GI Diarrhoea) } \\
\text { PROMIS Scale 9a (GI Constipation) } \\
\text { PROMIS Scale 13a (GI Gas and Bloating) } \\
\text { Peeds Dyspepsia Questionnaire }\end{array}$ & $\begin{array}{l}\text { PedsQL GI Symptoms Scale (stomach pain and hurt; } \\
\text { discomfort when eating; heartburn and reflux; nausea and } \\
\text { vomiting; gas and bloating; constipation; blood in BM; } \\
\text { diarrhoea; worry about stomachaches; worry about BM)- } \\
\text { age 5-17 }\end{array}$ \\
\hline & General quality of life & \\
\hline & Euro Quality of Life & $\begin{array}{l}\text { Euro Quality of Life Youth-age 8-15 } \\
\quad \text { Euro Quality of Life } 5 \text { Level-age 16+ }\end{array}$ \\
\hline & Psychological & \\
\hline & $\begin{array}{l}\text { Patient Health Questionnaire } \\
\text { PROMIS-29 (Physical Function, Anxiety, Depression, } \\
\text { Fatigue, Sleep Disturbance, Pain Interference and }\end{array}$ & $\begin{array}{l}\text { PROMIS-25 (Physical Function Mobility; Anxiety; } \\
\text { Depressive Symptoms; Fatigue; Peer Relations; Pain } \\
\text { Interference)-age 5-17 }\end{array}$ \\
\hline & Intensity) & Brief Resiliency Survey-age 12-17 \\
\hline & - GAD-7 & Pain Catastrophising Scale-age 8-17 \\
\hline & $\begin{array}{l}\text { Perceived Stress Scale } \\
\text { Adverse Childhood Experiences }\end{array}$ & Revised Child Anxiety and Depression Scale-age 6-17 \\
\hline & $\begin{array}{l}\text { Brief Resiliency Survey } \\
\text { Pain Catastrophising Scale }\end{array}$ & \\
\hline & Productivity & \\
\hline & Work Productivity and Activity Index & \\
\hline & Diet & \\
\hline & $\begin{array}{l}\text { Food Frequency Questionnaire } \\
\text { FODMAP Questionnaire }\end{array}$ & $\begin{array}{l}\text { Food Frequency Questionnaire } \\
\text { FODMAP Questionnaire }\end{array}$ \\
\hline
\end{tabular}

BM, bowel movement; CD, Crohn's disease; FODMAP, fermentable oligo-di-monosacharides and polyols; GI, gastrointestinal; IBD, inflammatory bowel disease; IBS, irritable bowel syndrome; PCDAI, Pediatric Crohn's Disease Activity Index; PedsQL, Pediatric Quality of Life Inventory; PROMIS, Patient-Reported Outcomes Measurement Information System; PUCAI, Paediatric Ulcerative Colitis Activity Index; UC, ulcerative colitis.

computer using an email link. The user questionnaires are available in the REDCap platform stored at a central database collection centre, PHRI, at McMaster University. Study staff will review surveys within 2 weeks of receipt and highlight any missing answers that suggest a problem in completing the survey. These issues will be discussed with the principal investigator, site lead and study team. The staff contact participants up to three times by phone email or in person at a regular study visit to remind them to complete questionnaires and to acquire answers to missing items.

\section{Patient and public involvement}

The research proposed in IMAGINE was informed by patients. Patient perspectives were sought in identifying priorities for strategic research funding opportunities. Diet, researching the gut microbiome and mental health were initially identified as key priorities through this process. Through the Crohn's and Colitis Canada (CCC) 'Gutsy Learning Series', these priorities were further defined with 289 lay participants in person and online with IBD who gave feedback on what they felt were the most important research topics, identifying faecal transplants, diet and mental health as key priorities. Furthermore, through a workshop organised by the Canadian Digestive Health Foundation (CDHF) prior to this IMAGINE SPOR application, a key message was that patients with IBS are concerned about using current pharmacological therapies to relieve their symptoms and prefer approaches that correct the imbalances they perceive to be the root cause of their disorder rather than taking drugs. Patients with IBS are concerned with the long-term use of powerful and sometimes expensive agents and would like more exploration of the factors that are driving the disease. One of the major areas this patient group wanted more research on was the role of diet and also the role that gut bacteria (and probiotics) play in driving IBS. There is remarkable congruence in the research priorities of the patient communities with IBD and IBS. The IMAGINE SPOR proposal was informed by these priorities and seeks to address them by exploring the diet-gut microbiomerelationship and how this influences GI and mental symptoms of IBS and IBD.

IMAGINE patient research partners were involved in the development of the MAGIC demographic questionnaire. They also served to pilot test the online questionnaires and provide feedback on user experience and feasibility.

IMAGINE patient partners have been directly involved with study design and recruitment of subjects in IMAGINE and will support capacity development for patient engagement more broadly. In-person interviews are being conducted by our patient partners to identify strategies 
to improve recruitment and retention rates. Also, this network of patient-engaged researchers is communicating knowledge derived from the study to healthcare professionals, policymakers and other patients.

A key component of the IMAGINE research programme is developing capacity for patient engagement, patient preferences for informing treatment choices and working with our patient partners to improve our recruitment rates and long-term retention of IMAGINE participants. We have a unique opportunity to integrate patient engagement as recommended by SPOR by leveraging our innovative Patient and Community Engagement Research (PaCER) programme based at the University of Calgary. ${ }^{25}{ }^{26}$ PaCER is designed to promote new roles for patients and family members in healthcare and health culture through engagement in research. PaCER provides opportunities for patients to be involved in the development and conduct of research designed to affect the lives of patients living with IBD and IBS. Involving patients and families in research is an opportunity to increase the capacity to anticipate problems, manage their condition as a partner in their healthcare team, and to support other patients and families.

\section{DATA MONITORING AND ETHICS}

This is an observational study with no intervention mandated by the protocol and so there is no external data safety and monitoring board. Research ethics approval has been obtained for all 15 sites involved in the study. The protocol was approved on 31 May 2017 with an approval number 2017-3000-GRA with the last amendment to date being on 25 June 2019 . The study was prospectively registered on 27 April 2017 (ClinicalTrials.gov Identifier: NCT03131414) and this was last updated on 7 March 2019. Recruitment commenced in October 2017.

\section{SAMPLE SIZE AND ANALYSES}

\section{Sample size}

UC, CD and IBS cases will be analysed. A sample size of 2000 cases in each disease group and 2000 healthy controls will have $90 \%$ power to detect a probability of 0.547 that an observed abundance in the disease group is more than the observed abundance in the control group using a Wilcoxon (Mann-Whitney) rank-sum test with a 0.001 two-sided significance level (to adjust for multiple testing). This sample size also assumes $20 \%$ data dropout. The probability of 0.547 was derived from 75 IBD cases that we have obtained from pilot studies. ${ }^{27}$

\section{Analyses}

Primary and secondary aims will be evaluated through multivariate logistic regression with further details regarding how diet, microbiome, metabolomics and genetics will be analysed below.

\section{Diet analyses}

We will use principal component analysis to derive dietary patterns from self-reported semi-quantitative food-frequency questionnaires (FFQs), and understand the differences in dietary patterns across participants with UC, CD, IBS and healthy controls; and for active versus non-active disease within each disease group, as outlined in the primary and secondary aims. Briefly, the purpose of principal component analysis (PCA) and/or machine learning is to reduce large and complex high-dimensional data into fewer dimensions-in this case comprehensive FFQ data (containing up to 150 items or more) is reduced to two or three dietary patterns (ie, foods commonly consumed together) that explain the greatest amount of dietary variability within the reported eating habits of the cohort. ${ }^{28}$ The number of dietary patterns to be retained for subsequent analysis will be based on visual inspection of Scree plots in conjunction with eigenvalues, and principal component interpretability. ${ }^{29}$ To account for differences in total energy intake between participants, dietary pattern scores will be adjusted to the mean total population caloric intake using the residual method. ${ }^{28}$ Associations between a participant's adherence to a specific PCA-derived dietary pattern (eg, Western or plant-based) and UC, CD, IBS and its disease activity will be quantified using logistic regression (case vs control) with appropriate adjustment for covariates (eg, BMI, age, sex, and so on). For machine learning, dietary patterns will be derived using unsupervised methods that require little to no input or direction by the researcher. The use of both PCA and unsupervised machine-learning methods will allow for validation of the derived diet patterns. Our experience suggests that the PCA and machine-learning derided patterns will be largely similar. ${ }^{30}$

In an exploratory analysis, of particular interest for the IBS group, we will collect data using a supplementary fermentable oligo-di-monosacharides and polyols (FODMAP) Questionnaire to capture foods rich in: (a) oligosaccharides, including fructans and galactooligosaccharides; (b) disaccharides, including lactose; (c) monosaccharides, including fructose; (d) polyols. These data will be used to better understand 'trigger foods' or dietary components that are likely to produce symptoms in participants with IBS.

\section{Microbiome processing and analyses}

All stool samples will be processed in one lab for consistency. Frozen samples sent from each site will be thawed on ice in an anaerobic environment, mixed thoroughly with a sterile spatula. Two aliquots of $0.3 \mathrm{~g}$ will be transferred to DNA extraction buffer for molecular analysis. Three aliquots of $1.8 \mathrm{~mL}$ will be biobanked at $-80^{\circ} \mathrm{C}$. DNA will be extracted using established methods. ${ }^{31}$ Total bacterial load will be measured by quantitative PCR of the $16 \mathrm{~S}$ rRNA gene. Microbial community profiling will be carried out by amplification and paired-end Illumina sequencing of the v3-v4 region of the 16S rRNA gene for bacteria ${ }^{3132}$ and the Internal Transcribed Region of the ribosomal genes for fungi. ${ }^{33}$ Microbiome profiles will be processed through in-house bioinformatic pipelines ${ }^{34}$ incorporating dada $2^{35}$ to generate amplicon sequence variants. 
As sequencing and library construction costs decrease, it will be feasible to carry out shotgun metagenomics on a significant portion of the stool samples. We aim to reduce costs so it is possible to perform this on all samples but if this is not possible, we will perform shotgun metagenomics on at least $20 \%$ of randomly selected samples from each group. Metagenomic sequencing libraries will be constructed using NEBNext Ultra DNA Library kits with modifications to reduce reaction volume. Libraries will be sequenced to $\sim 15000000$ reads per sample (150nt paired-end) on an Illumina NovaSeq. After filtering and trimming for sequence quality and primer removal, DeconSeq ${ }^{36}$ will be performed on the remaining reads in order to remove reads of human DNA. Genes and functional predictions and comparisons across sample groups will be computed using HUMAnN $2^{37}$ and MetaPHIAn ${ }^{38}$ for functional microbiome analysis.

Microbiome analysis will include $\alpha$-diversity metrics for each sample (observed species, chao1, Shannon diversity) and $\beta$-diversity measures will be used to compare diversity between samples. For the latter, centred log-ratio transformation of the read count data will be carried out to account for the compositional nature of microbiome data ${ }^{39}$ and visualised using Aitchison principal-component analysis. ${ }^{40}$ Statistical analyses will be carried out in R using PhyloSeq ${ }^{41}$ and ALDEx $2{ }^{42}$ in R. Using generalised linear mixed models, we will identify microbial taxa and/or genes associated with disease phenotypes/progression, response to treatment, genotype, diet and other measured parameters. The large number of samples will also allow for application of machine learning methods such as random forest and support vector machine methods. ${ }^{43} 44$

\section{Metabolomics processing and analyses}

Urinary metabolomic profiles will be determined and analysed together with microbial and dietary profiles to identify relationships and associations with disease status and clinical phenotypes/response to therapies. Urinary metabolic profiles will be analysed by ${ }^{1} \mathrm{H}-\mathrm{NMR}$ on a 4-channel Varian INOVA $600 \mathrm{MHz}$ NMR spectrometer. Assignment of endogenous urinary metabolites will be done using Chenomx NMR Suite V.8.5 (Chenomx, Edmonton, Canada) and online databases (HMDB). ${ }^{45}$ Metabolite concentrations will be $\log _{10}$ transformed to normalise data prior to statistical analyses. Metabolome association study analyses will be done using multiple linear regression models in the $\mathrm{R}$ Project for Statistical Computing (R program). Projection-based PCA, partial least-squares discriminant analysis, orthogonal partialleast squares analysis will be performed using $\mathrm{R}$ program.

\section{Genetic analyses}

In terms of genetic analyses, genomic DNA samples will be tested using two different approaches: (1) genome-wide genotyping to capture common genetic variation and enable genome-wide association studies and (2) whole exome sequencing will primarily be used to capture rare genetic variation and identify non-synonymous coding variants as potential causal variants. Both datasets will be used to identify genetic risk variants associated with disease status or clinical phenotypes/outcomes detailed above (eg, response to therapy); both as previously described. ${ }^{46-49}$ These data will also be used to impute the genetic variation at the highly polymorphic human leucocyte antigens and killer cell immunoglobulin-like receptor genes, ${ }^{5051}$ as these are key determinants of the host's immune response and genetic risk factors for many inflammatory diseases.

For the statistical analyses, following rigorous quality control of the genotype/sequence data, whole genome imputation of the dataset using a relevant public reference panel (eg, 1000 Genomes, Haplotype Reference Panel, TOPMed, and so on $)^{52} 53$ will be performed followed by principal components analysis (PCA). Principal components will be tested for phenotype association (using logistic regression with study indicator variables included as covariates) and evaluated for their impact on the genome-wide test statistics using $\lambda$ (the genomic control inflation factor based on the median $\chi 2)$ after genome-wide association of the specified principal component. Association testing as well as binary and linear genotype-phenotype analyses will be done with PLINK and multinomial and ordinal regression analyses with a custom program, Trinculo. Survival analysis and risk prediction will be done with $\mathrm{R}$ using the packages 'survival' and 'Mangrove', respectively. For integrated biomarker discovery, ${ }^{54}$ this genetic data will also be integrated with other biomarker data generated from the various IMAGINE platforms, in order to select those that estimate a large association with clinical outcomes (eg, response to therapy), in order to create the best subset of predictors. Variable selection will be based on mathematical criteria for model selection, that is, the Bayesian Information Criteria (BIC), and expert a priori (eg, clinical knowledge, preliminary evidence). The selection of the model will be in the context of logistic regression, using the candidate biomarkers as covariates and drug response (positive or negative) as the outcome. The BIC has been proven to lead to less overfitting of the model to data compared with other less conservative approaches. ${ }^{55}$ This will reduce type-I errors and lead to increased robustness of the results.

\section{Subgroup analyses}

There are a number of subgroup analyses planned. In particular, we will analyse the primary and secondary outcomes by sex. We will strive to ensure representative enrolment of men and women with a wide range of life experience and at different life stages. When women are surveyed or otherwise evaluated, we will take note of past, anticipated and ongoing pregnancies, obtain a menstrual history and use instruments that are sensitive to the influence of gender on outcomes.

The paediatric population (IBD cases 4-18 years of age) is also an important group to study. All the primary and secondary outcomes described above will also be 
evaluated specifically in the paediatric population. This includes predictors of success and failure of therapy for IBD. Children developing IBD are predominantly treated from the time of first presentation at academic centres rather than in community practice. Hence, the paediatric collaborators in this proposal offer access to the broad spectrum of IBD, including prior to alteration of the microbiome by any therapy. We will evaluate predictors of success and failure of these therapies for IBD over time in the paediatric population.

We will evaluate subtypes of IBS; IBS-C, IBS-D and IBS-M. We will assess the microbiome, metabolomics, genetic, demographic and dietary predictors of response to therapies for IBS. Predictors of response to a low FODMAPs diet will help inform a randomised controlled trial (RCT) of low FODMAPs diet in IBS, and in particular we will evaluate whether responders are more likely to initially be taking a diet that is very rich in FODMAPs and how this response relates to their microbiome compositional and functional changes, affected by diets. A subgroup analysis will be performed of those between the ages of 13 and 17 years compared with adult patients. We will also evaluate those with stable coeliac disease and compare results to those without this disorder.

\section{DISSEMINATION}

Our approach to dissemination involves developing evidence-based knowledge translation initiatives for research that is ready for prime time-for each of category of our three research partners (patient, clinicians and policymakers). For patients, we plan to create a 'white label' version of the McMaster Optimal Ageing Portal (https://www.mcmasteroptimalaging.org) that focuses specifically on supporting self-management and more generally informed decision-making for GI disease. This involves (1) identifying existing Evidence Summaries and preparing new ones to provide patients with the key messages from scientific research (typically high-quality systematic reviews) that are ready to be acted on; (2) identifying existing Web Resource Ratings and preparing new ones to help patients identify the free health resources on the internet that are based on scientific research; (3) identifying existing Blog Posts and preparing new ones that provide commentaries for patients about on what the scientific research on a topic actually means and on why good science matters; and (4) identifying existing patient decision aids to help patients (and clinicians working with patients) to engage in shared decision-making.

We will work with network partners (eg, CDHF and CCC) to determine the best online platform for patientfocused products, to develop inclusion criteria, and to ensure that their online resources focused on GI disease meet the high standard necessary to be captured and ranked highly in the Web Resource Ratings. We plan to prepare 52 new Evidence Summaries, 24 Blog Posts and 400 Web Resources Ratings for the 'white label' portal. We will also be working with network partners to ensure high rates of use of the white-label, GI disease-focused content site for patients.

The patients, family members and caregivers who live with the chronic conditions covered by the IMAGINE Network will be able to use the portal to find information that aids them in managing their conditions and making related health decisions. The content produced by the McMaster Optimal Ageing Portal has been shown to be effective in informing health consumers of quality heath information related to ageing but not in other domains. We plan to conduct an RCT (which will be detailed in a separate protocol) to assess how the online resources for patients, provided through the white label website, changes patient behaviour in regards to using information and making evidence-informed health decisions.

For clinicians, we will follow an approach that emerged from a comprehensive review of the literature on scaling up effective clinical interventions (summarised in an evidence brief) ${ }^{56}$ and a stakeholder dialogue involving the key policymakers, stakeholders and researchers focused on supporting scale-up of effective clinical practices in Ontario (summarised in a dialogue summary) ${ }^{57}$ : (1) supporting dynamic efforts to identify GI diseaserelated clinical practices to be optimised and the causes of underlying problems, using both empirical approaches like systematic reviews and theoretical approaches like the Behaviour Change Wheel and the Theoretical Domains Framework; (2) using rigorous processes to select and implement approaches to optimising clinical practices that address the underlying causes of problems (eg, audit and feedback, financial incentives); and (3) monitoring, evaluating and reviewing the approaches selected to optimise clinical practices. We plan to support two approaches: (1) prioritising clinical interventions to be scaled up by engaging network members using an explicit process; and (2) scaling up effective clinical interventions by developing and executing a scale-up plan, both of which will build on what has been learnt from related work at the McMaster Health Forum, and will rely on the frequent engagement of key members of the IMAGINE SPOR Network in doing so.

For policymakers, we will use our tried and tested approach to supporting evidence-informed policymaking, which means: (1) preparing an evidence brief on a pressing need for scale-up and the factors hindering that achievement (eg, nurses' scope of practice, existing financial incentives, lack of multidisciplinary teams), options for scaling up and key implementation considerations (which includes an equity lens brought to bear on particularly vulnerable groups); (2) convening a stakeholder dialogue that brings together key policymakers, patient and clinical leaders and researchers who can consider the research evidence alongside the other factors that drive decision-making; and (3) preparing a dialogue summary, disseminating the evidence brief and dialogue summary, conducting personalised briefings to senior leaders in the system, and supporting their efforts to act on what they learnt. 
We will enhance this approach by convening citizen panels to capture the insights and values of a diverse group of citizens, with different types of lived experience with the issue at hand, in a panel summary, the key messages of which would be included in the evidence brief informing the stakeholder dialogue. We plan to address two topics using this approach, one focused on reducing emergency-department usage in people with IBD, and one focused on framing the work led by IMAGINE in the context of rapid learning health systems for specific conditions (including those addressed by the IMAGINE Network). For each topic we will conduct consultations with a steering committee and key informants to define the terms of reference for evidence briefs, identify stakeholders and potential dialogue invitees, and review the outputs (briefs, summaries and evaluation reports).

\section{ARTICLE SUMMARY \\ Strengths}

Studies that have evaluated the microbiome in $\mathrm{IBS}^{18}$ and $\mathrm{IBD}^{19}$ have been small and underpowered. These studies usually did not evaluate disease phenotype in detail and have not assessed diet, ${ }^{18} 19$ which can be an important effect modifier. ${ }^{58}$ This will be the largest observational study published to date that is sufficiently powered to evaluate the microbiome in IBS and IBD. All relevant confounding factors and effect modifiers will be captured and followed over time and longitudinally, which will allow a better understanding of what drives exacerbations of both IBS and IBD given that these are chronic relapsing and remitting diseases. There is also the possibility of pooling data with similar cohorts to provide more robust data on microbiome changes over time in these diseases. ${ }^{59}$ The other strength of the study is the multidisciplinary team that makes up the IMAGINE Network. In particular, the collaboration between psychiatry and gastroenterology allows a careful evaluation of gut brain connections ${ }^{17}$ and in particular how the gut microbiome ${ }^{60}$ may impact on anxiety and depression in patients with IBD and IBS. Another key component of the multidisciplinary team is the level of patient engagement throughout the proposal. This is a Canadian Institute of Health Research funded grant through the SPOR initiative mandating that priorities are set by patients and they have input into study design. To date, we have 19 patient partners as part of the IMAGINE Network and many are involved in the MAGIC cohort study. This strengthens the research, making it more patient focused, and supports knowledge translation of the findings to patients.

\section{Limitations}

This study is observational so any associations found may not relate to the causes of IBS and IBD. We will rigorously control for confounding factors and but cannot control for unknown confounders and so cannot draw causal inferences from the data. The MAGIC study will therefore be hypothesis generating and any data relating to the microbiome or diet are likely to need confirmation in RCTs. The IMAGINE Network is committed to develop RCTs to further investigate any promising findings from the MAGIC cohort study. This is the largest study evaluating IBS and IBD, but the data that will be collected are enormous and so any results related to the secondary outcomes of the study need to be interpreted with caution. The adjustment for multiple testing is for the primary outcomes only and given the number of other outcomes that can be evaluated with the data that are generated, it is important to realise that any positive results from the secondary outcomes could be a chance finding related to multiple testing. We will highlight this when reporting the data of more exploratory outcomes being studied. Despite these caveats, the MAGIC Study will provide valuable insight into the aetiology of IBS and IBD as well as associated psychiatric disorders. Data from this study will also provide strategies for personalised medicine approaches to manage these diseases more effectively.

Author affiliations

${ }^{1}$ Medicine, McMaster University Faculty of Health Sciences, Hamilton, Ontario, Canada

${ }^{2}$ Medicine, University of Calgary, Calgary, Alberta, Canada

${ }^{3}$ Gastroenterology, University of Manitoba, Winnipeg, Manitoba, Canada

${ }^{4}$ Medicine, Queen's University, Kingston, Ontario, Canada

${ }^{5}$ Medicine, University of Alberta, Edmonton, Ontario, Canada

${ }^{6}$ McMaster University Faculty of Health Sciences, Hamilton, Alberta, Canada

${ }^{7}$ Universite de Montreal, Montreal, Ontario, Canada

${ }^{8}$ Faculty of Medicine and Dentistry, University of Alberta, Edmonton, Alberta, Canada

${ }^{9}$ Division of Gastroenterology, McMaster University Faculty of Health Sciences,

Hamilton, Ontario, Canada

${ }^{10}$ Department of Clinical Epidemiology and Biostatistics, McMaster University

Faculty of Health Sciences, Hamilton, Ontario, Canada

${ }^{11}$ Dalhousie University, Halifax, Nova Scotia, Canada

${ }^{12}$ Mount Sinai Hospital, Toronto, Ontario, Canada

${ }^{13}$ Population Health Research Institute, McMaster University Faculty of Health Sciences, Hamilton, Ontario, Canada

${ }^{14}$ Department of Community Health Sciences, University of Calgary, Calgary, Alberta, Canada

${ }^{15} \mathrm{PaCER}$ Innovates, University of Calgary, Calgary, Alberta, Canada

Acknowledgements We are grateful to Glenda MacQueen for all the support she gave to the IMAGINE Network and the MAGIC Study. Her advice was invaluable throughout this project and she will be missed by all of us. We are grateful to all our patient partners and everyone within the IMAGINE Network.

Contributors PM, GM, CNB, SV, PB, KLM, MS, JDR, LAD, EV, RJdS, A0, LT, JL, JC, $\mathrm{DAM}, \mathrm{SZ}$ and $\mathrm{AF}$ have contributed to the conception and design of the protocol and obtained funding. PM, MS, JDR, KLM and RJdS provided statistical support. JDR led the genetic, MS the microbiome, KLM the metabolomic, GM the psychiatric, CNB the inflammatory bowel disease, PB and SV the irritable bowel syndrome, LAD, EV and RJdS the diet, $A 0$ the paediatric, JL the knowledge translation, DAM and SV the patient engagement and JC the data management portions of the protocol. PM and $A F$ constructed the first draft of the article which was significantly revised by all other authors. CNB, SV, PB, KLM, MS, JDR, LAD, EV, RJdS, AO, LT, JL, JC, DAM and SZ have provided informatics support, given critical revisions to the manuscript and approved the final version of the manuscript submitted for publication. PM acts as the guarantor for the article.

Funding The IMAGINE Network is supported by a grant from the Canadian Institute of Health Research (Funding Reference Number: RN279389-35803) with funding from several partners. Funding was matched by McMaster University, University of Calgary, University of Alberta, Queen's University, Dalhousie University, Montreal Heart Institute Research Centre, Takeda Pharmaceutical Company, Allergan Incorporated, Alberta Innovates, Research Manitoba, Crohn's and Colitis Canada. 
Competing interests PM holds the Audrey Campbell Chair in Ulcerative Colitis Research. PM, PB and AF have no conflicts of interest. CNB is supported in part by the Bingham Chair in Gastroenterology. He is on Advisory Boards for AbbVie Canada, Janssen Canada, Takeda Canada, Pfizer Canada. He is a consultant for Mylan Pharmaceuticals. He is receiving educational grants from AbbVie Canada, Pfizer Canada, Shire Canada, Takeda Canada, Janssen Canada. Speaker's panel for AbbVie Canada, Janssen Canada, Takeda Canada, and Medtronic Canada. Received research funding from AbbVie Canada. JDR receives research funding from Pfizer. LAD is on the Advisory Boards for Janssen Canada, AbbVie Canada, Pfizer Canada and Takeda Canada. DAM holds a Canada Research Chair (2008-2018) and the Arthur J.E. Child Chair and receives travel reimbursement through Illumina for meetings of the Global Economics and Evaluation of Clinical Genomics Sequencing Working Group. SV was supported by an educational grant from Allergan. SZ is a patient research partner and received a grant from Takeda Canada.

\section{Patient consent for publication Not required}

Provenance and peer review Not commissioned; externally peer reviewed.

Supplemental material This content has been supplied by the author(s). It has not been vetted by BMJ Publishing Group Limited (BMJ) and may not have been peer-reviewed. Any opinions or recommendations discussed are solely those of the author(s) and are not endorsed by BMJ. BMJ disclaims all liability and responsibility arising from any reliance placed on the content. Where the content includes any translated material, BMJ does not warrant the accuracy and reliability of the translations (including but not limited to local regulations, clinical guidelines, terminology, drug names and drug dosages), and is not responsible for any error and/or omissions arising from translation and adaptation or otherwise.

Open access This is an open access article distributed in accordance with the Creative Commons Attribution Non Commercial (CC BY-NC 4.0) license, which permits others to distribute, remix, adapt, build upon this work non-commercially, and license their derivative works on different terms, provided the original work is properly cited, appropriate credit is given, any changes made indicated, and the use is non-commercial. See: http://creativecommons.org/licenses/by-nc/4.0/.

\section{ORCID iDs}

Paul Moayyedi http://orcid.org/0000-0002-3616-9292

Jennifer Cunningham http://orcid.org/0000-0001-8137-6779

Deborah A Marshall http://orcid.org/0000-0002-8467-8008

\section{REFERENCES}

1 Thompson WG, Irvine EJ, Pare P, et al. Functional gastrointestinal disease in Canada. Dig Dis Sci 2002;47:225-35.

2 Sperber AD, Bangdiwala SI, Drossman DA, et al. Worldwide prevalence and burden of functional gastrointestinal disorders, results of Rome Foundation global study. Gastroenterology 2020:S0016-5085:30487-X.

$3 \mathrm{Ng} \mathrm{SC}$, Shi HY, Hamidi N, et al. Worldwide incidence and prevalence of inflammatory bowel disease in the 21 st century: a systematic review of population-based studies. Lancet 2018;390:2769-78.

4 Brandt LJ, Chey WD, et al. An evidence-based position statement on the management of irritable bowel syndrome. Am J Gastroenterol 2009;104 Suppl 1:S8-35.

5 Talley NJ, Abreu MT, Achkar J-P, et al. An evidence-based systematic review on medical therapies for inflammatory bowel disease. $A m \mathrm{~J}$ Gastroenterol 2011;106 Suppl 1:S2-25

6 Gralnek IM, Hays RD, Kilbourne A, et al. The impact of irritable bowel syndrome on health-related quality of life. Gastroenterology 2000;119:654-60.

7 Casellas F, López-Vivancos J, Badia X, et al. Influence of inflammatory bowel disease on different dimensions of quality of life. Eur J Gastroenterol Hepatol 2001;13:567-72.

8 Bernstein CN, Loftus EV, Ng SC, et al. Hospitalisations and surgery in Crohn's disease. Gut 2012;61:622-9.

9 Peery AF, Crockett SD, Barritt AS, et al. Burden of gastrointestinal, liver, and pancreatic diseases in the United States. Gastroenterology 2015;149:1731-41.

10 Lee C, Doo E, Choi JM, et al. The increased level of depression and anxiety in irritable bowel syndrome patients compared with healthy controls: systematic review and meta-analysis. J Neurogastroenterol Motil 2017;23:349-62.

11 Mikocka-Walus A, Knowles SR, Keefer L, et al. Controversies revisited: a systematic review of the comorbidity of depression and anxiety with inflammatory bowel diseases. Inflamm Bowel Dis 2016;22:752-62.
12 Jones R, Latinovic R, Charlton J, et al. Physical and psychological co-morbidity in irritable bowel syndrome: a matched cohort study using the general practice research database. Aliment Pharmacol Ther 2006;24:879-86.

13 Roy-Byrne PP, Davidson KW, Kessler RC, et al. Anxiety disorders and comorbid medical illness. Gen Hosp Psychiatry 2008;30:208-25.

14 Ford AC, Moayyedi P, Lacy BE, et al. American College of gastroenterology monograph on the management of irritable bowe syndrome and chronic idiopathic constipation. Am J Gastroenterol 2014;109 Suppl 1:S2-26.

15 Moayyedi P, Simrén M, Bercik P. Evidence-Based and mechanistic insights into exclusion diets for IBS. Nat Rev Gastroenterol Hepatol 2020;17:406-13.

16 Moayyedi P. Update on fecal microbiota transplantation in patients with inflammatory bowel disease. Gastroenterol Hepatol 2018;14:319-22.

17 MacQueen G, Surette M, Moayyedi P. The gut microbiota and psychiatric illness. J Psychiatry Neurosci 2017;42:75-7.

18 Pittayanon R, Lau JT, Yuan Y, et al. Gut microbiota in patients with irritable bowel syndrome-a systematic review. Gastroenterology 2019;157:97-108.

19 Pittayanon R, Lau JT, Yuan Y, et al. Gut microbiota in patients with inflammatory bowel disease - a systematic review. Gastroenterology 2020;158:930-46.

20 IMAGINE. Will transform the management of IBD and IBS and associated mental health issues with these disorders. Available: www.imaginespor.com

21 Palsson OS, Whitehead WE, van Tilburg MAL, et al. Development and validation of the Rome IV diagnostic questionnaire for adults. Gastroenterology 2016;150:1481-91.

22 Satsangi J, Silverberg MS, Vermeire S, et al. The Montreal classification of inflammatory bowel disease: controversies, consensus, and implications. Gut 2006;55:749-53.

23 Levine A, Griffiths A, Markowitz J, et al. Pediatric modification of the Montreal classification for inflammatory bowel disease: the Paris classification. Inflamm Bowel Dis 2011;17:1314-21.

24 Ruemmele FM, Hyams JS, Otley A, et al. Outcome measures for clinical trials in paediatric IBD: an evidence-based, expert-driven practical statement paper of the paediatric ECCO Committee. Gut 2015;64:438-46.

25 Shklarov S, Marshall DA, Wasylak T, et al. "Part of the Team": Mapping the outcomes of training patients for new roles in health research and planning. Health Expect 2017;20:1428-36.

26 Marlett N, Shklarov S, Marshall D, et al. Building new roles and relationships in research: a model of patient engagement research Qual Life Res 2015;24:1057-67.

27 Moayyedi P, Surette MG, Kim PT, et al. Fecal microbiota transplantation induces remission in patients with active ulcerative colitis in a randomized controlled trial. Gastroenterology 2015;149:102-9

$28 \mathrm{Hu}$ FB. Dietary pattern analysis: a new direction in nutritional epidemiology. Curr Opin Lipidol 2002;13:3-9.

$29 \mathrm{Hu}$ FB, Rimm EB, Stampfer MJ, et al. Prospective study of major dietary patterns and risk of coronary heart disease in men. Am J Clin Nutr 2000;72:912-2.

30 Northstone K, Ness AR, Emmett PM, et al. Adjusting for energy intake in dietary pattern investigations using principal components analysis. Eur J Clin Nutr 2008;62:931-8.

31 Lau JT, Whelan FJ, Herath I, et al. Capturing the diversity of the human gut microbiota through culture-enriched molecular profiling. Genome Med 2016;8:72.

32 Woo TE, Lim R, Heirali AA, et al. A longitudinal characterization of the non-cystic fibrosis bronchiectasis airway microbiome. Sci Rep 2019;9:6871.

33 Xu J. Fungal DNA barcoding. Genome 2016;59:913-32.

34 Whelan FJ, Surette MG. A comprehensive evaluation of the sl1p pipeline for $16 \mathrm{~S}$ rRNA gene sequencing analysis. Microbiome 2017;5:100

35 Callahan BJ, McMurdie PJ, Rosen MJ, et al. DADA2: high-resolution sample inference from Illumina amplicon data. Nat Methods 2016;13:581-3.

36 Schmieder R, Edwards R. Fast identification and removal of sequence contamination from genomic and metagenomic datasets. PLoS One 2011;6:e17288.

37 Franzosa EA, Mclver LJ, Rahnavard G, et al. Species-Level functional profiling of metagenomes and metatranscriptomes. Nat Methods 2018;15:962-8.

38 Segata N, Waldron L, Ballarini A, et al. Metagenomic microbial community profiling using unique clade-specific marker genes. Nat Methods 2012:9:811-4. 
39 Gloor GB, Macklaim JM, Pawlowsky-Glahn V, et al. Microbiome datasets are compositional: and this is not optional. Front Microbiol 2017;8:2224.

40 Martino C, Morton JT, Marotz CA, et al. A novel sparse compositional technique reveals microbial perturbations. mSystems 2019;4:e00016-19.

41 McMurdie PJ, Holmes S. phyloseq: an R package for reproducible interactive analysis and graphics of microbiome census data. PLOS One 2013;8:e61217.

42 Fernandes AD, Reid JN, Macklaim JM, et al. Unifying the analysis of high-throughput sequencing datasets: characterizing RNA-seq, 16S rRNA gene sequencing and selective growth experiments by compositional data analysis. Microbiome 2014;2:15

43 Qu K, Guo F, Liu X, et al. Application of machine learning in microbiology. Front Microbiol 2019;10:827.

44 Camacho DM, Collins KM, Powers RK, et al. Next-Generation machine learning for biological networks. Cell 2018;173:1581-92.

45 Wishart DS, Tzur D, Knox C, et al. HMDB: the human metabolome database. Nucleic Acids Res 2007;35:D521-6.

46 Anderson CA, Boucher G, Lees CW, et al. Meta-Analysis identifies 29 additional ulcerative colitis risk loci, increasing the number of confirmed associations to 47. Nat Genet 2011;43:246-52.

47 Rivas MA, Avila BE, Koskela J, et al. Insights into the genetic epidemiology of Crohn's and rare diseases in the Ashkenazi Jewish population. LoS Genet 2018;14:e1007329.

48 Huang $\mathrm{H}$, Fang $\mathrm{M}$, Jostins $\mathrm{L}$, et al. Fine-Mapping inflammatory bowel disease loci to single-variant resolution. Nature 2017;547:173-8.

49 Cleynen I, Boucher G, Jostins L, et al. Inherited determinants of Crohn's disease and ulcerative colitis phenotypes: a genetic association study. Lancet 2016;387:156-67.
50 Goyette P, Boucher G, Mallon D, et al. High-Density mapping of the $\mathrm{MHC}$ identifies a shared role for HLA-DRB1*01:03 in inflammatory bowel diseases and heterozygous advantage in ulcerative colitis. Nat Genet 2015;47:172-9.

51 Vukcevic D, Traherne JA, Næss S, et al. Imputation of Kir types from SNP variation data. Am J Hum Genet 2015;97:593-607.

52 Gourraud P-A, Khankhanian P, Cereb N, et al. Hla diversity in the 1000 genomes dataset. PLoS One 2014;9:e97282.

53 Das S, Abecasis GR, Browning BL. Genotype imputation from large reference panels. Annu Rev Genomics Hum Genet 2018;19:73-96.

54 Ivison S, Des Rosiers C, Lesage S, et al. Biomarker-Guided stratification of autoimmune patients for biologic therapy. Curr Opin Immunol 2017:49:56-63.

55 Gao X, Song PX-K. Composite likelihood Bayesian information criteria for model selection in high-dimensional data. J Am Stat Assoc 2010;105:1531-40.

56 Lavis JN, Wilson MG, Grimshaw JM. Evidence brief: optimizing clinical practice in Ontario based on data, evidence and guidelines. Hamilton, Canada: McMaster Health Forum, 2015.

57 Wilson MG, Lavis JN. Dialogue summary: optimizing clinical practice in Ontario based on data, evidence and guidelines. Hamilton, Canada: McMaster Health Forum, 2015.

58 Mclntosh K, Reed DE, Schneider T, et al. FODMAPs alter symptoms and the metabolome of patients with IBS: a randomised controlled trial. Gut 2017;66:1241-51.

59 Yilmaz $B$, Juillerat $P$, Øyås $\mathrm{O}$, et al. Microbial network disturbances in relapsing refractory Crohn's disease. Nat Med 2019;25:323-36 https://doi.org/

60 Bercik P, Collins SM, Verdu EF. Microbes and the gut-brain axis. Neurogastroenterol Motil 2012;24:405-13. 